\title{
COMMUTING OF TOEPLITZ OPERATORS ON THE BERGMAN SPACES OF THE BIDISC
}

\author{
YUFENG Lu
}

In this paper we describe when two Toeplitz operators $T_{f}$ and $T_{g}$ on the Bergman space of the bidisc commute, where $f=f_{1}+\overline{f_{2}}, g=g_{1}+\overline{g_{2}}, f_{i}, g_{i} \in H^{\infty}\left(D^{2}\right)(i=1,2)$.

\section{INTRODUCTION}

For a bounded domain $\Omega$ in $C^{n}$, let $d A$ denote the Lebesgue measure on $\Omega$ normalised so that $\Omega$ has measure 1 . The Bergman space $L_{a}^{2}(\Omega)$ is the Hilbert space consisting of analytic functions on $\Omega$ that are also in $L^{2}(\Omega, d A)$. The norm $\|\cdot\|_{2}$ and the inner product $\langle$,$\rangle are those of the space L^{2}(\Omega, d A)$. In this paper let $\Omega$ denote the bidisc $D^{2}$. For $z \in \Omega$, the Bergman reproducing kernel is the function $K_{z} \in L_{a}^{2}(\Omega)$ such that

$$
f(z)=\left\langle f, K_{z}\right\rangle
$$

for every $f \in L_{a}^{2}(\Omega)$. The normalised Bergman reproducing kernel $k_{z}$ is the function $K_{z} /\left\|K_{z}\right\|_{2}$.

Let $P$ denote the orthogonal projection of $L^{2}(\Omega, d A)$ onto $L_{a}^{2}(\Omega)$. For a function $f \in L^{\infty}(\Omega, d A)$, the Toeplitz operator $T_{f}: L_{a}^{2}(\Omega) \rightarrow L_{a}^{2}(\Omega)$ is defined by

$$
T_{f}(g)=P(f g), g \in L_{a}^{2}(\Omega) .
$$

These are clearly bounded linear operators for every function $f \in L^{\infty}(\Omega)$. Note that if $f$ is a bounded analytic function on $\Omega$, then $T_{f}$ is simply the operator of multiplication by $f$ on $L_{a}^{2}(\Omega)$.

For $f \in L^{2}(\Omega, d A)$, we define $\tilde{f}$, the Berezin symbol of $f$, by

$$
\tilde{f}(z)=\left\langle f k_{z}, k_{z}\right\rangle=\int_{\Omega} f(w)\left|k_{z}(w)\right|^{2} d A(w), z \in \Omega .
$$

By making the change of variable $z=\varphi_{w}(u)$, where $\varphi_{z}(w)$ is the Möbius transformation on $\Omega$, we have

$$
\widetilde{f}=\int_{\Omega} f \circ \varphi_{z}(w) d A(w)
$$

Received 27th May, 2002

Copyright Clearance Centre, Inc. Serial-fee code: 0004-9727/02 \$A2.00+0.00. 
For the polydisk $D^{n}$, Möbius transformations are described in [4, Section 10.1].

The general problem that we are interested in is the following: when two Toeplitz operators commute, what is the relationship between their symbols? On the Hardy space of the circle, this question has been solved in [3]. On the Bergman space of the unit disk, partial results were obtained by Axler and Čučković [1] and Axler and Čučković and Rao [2]. On the Bergman space of several complex variables, the situation is more complicated. In this paper we give a partial result for the general problem on the Bergman space of the bidisc.

\section{Commutants of Toeplitz Operators on the Bidisc}

Following [6, Definition 2.1.1], we say $f \in C\left(D^{n}\right)$ is $n$-harmonic if $f$ is harmonic in each variable separately, that is,

$$
\Delta_{1} f=\Delta_{2} f=\cdots=\Delta_{n} f=0 .
$$

Here variable means complex variable. If $z_{j}=x_{j}+i y_{j}$,

$$
\Delta_{j} f=4 \frac{\partial}{\partial \bar{z}}\left(\frac{\partial f}{\partial z}\right)=\frac{\partial^{2}}{\partial x_{j}^{2}}+\frac{\partial^{2}}{\partial y_{j}^{2}}
$$

Since the harmonic functions are those for which $\sum \Delta_{j}=0$, every $n$-harmonic function is harmonic. The two classes coincide if and only if $n=1$.

In this section, we describe when $T_{f}$ and $T_{g}$ defined on the Bergman space of the bidisc commute, where $f=f_{1}+\overline{f_{2}}, g=g_{1}+\overline{g_{2}}, f_{i}, g_{i} \in H^{\infty}\left(D^{2}\right)$.

We use $f\left(z_{1}\right)$ (or $f\left(z_{2}\right)$ ) to denote the function $f\left(z_{1}, z_{2}\right)$ on $D^{2}$ which depends only $z_{1}$ (or $z_{2}$ ).For a function $f\left(z_{1}\right)$, we set $\frac{\partial f}{\partial z_{1}}=f^{\prime}$. In this section, $c$ always denotes a constant, and it may be different in different places. Suppose $f=f_{1}+\overline{f_{2}}, g=g_{1}+\overline{g_{2}}$, $f_{i}, g_{i}(i=1,2) \in H^{\infty}\left(D^{2}\right)$. We study this problem for the following four types of functions. TYPE 1. $f, g$ have the following form:

$$
f=f_{1}\left(z_{2}\right)+\overline{f_{2}}, g=\overline{g_{2}\left(z_{1}\right)}+c
$$

or

$$
f=f_{1}(z)+\overline{f_{2}\left(z_{2}\right)}, g=g_{1}\left(z_{1}\right)+c .
$$

Interchanging $f$ and $g$ or $z_{1}$ and $z_{2}$,we can obtain all of Type 1 functions. For example, $f=f_{2}\left(z_{1}\right)+\overline{f_{2}}, g=\overline{g_{2}\left(z_{2}\right)}+c$ is Type 1 , too.

TYPE 2. $f, g$ have the following form.

$$
f=f_{1}\left(z_{2}\right)+\overline{f_{2}\left(z_{1}\right)}, g=g_{1}\left(z_{2}\right)+\overline{g_{2}\left(z_{1}\right)},
$$


or

$$
f=f_{1}\left(z_{1}\right)+\overline{f_{2}\left(z_{2}\right)}, g=g_{1}\left(z_{1}\right)+\overline{g_{2}\left(z_{2}\right)}
$$

TYPE 3. $f, g$ satisfy the following condition. There exist constants $c, c_{1}$, and an analytic function $a$ such that

$$
f_{1}=c g_{1}\left(z_{2}\right)+c_{0}, f_{2}=\bar{c} g_{2}(z)+a\left(z_{1}\right) .
$$

Interchanging $f$ and $g$ or $z_{1}$ and $z_{2}$, we can obtain all of Type 3 functions. For example, $f_{1}=c g_{1}(z)+a\left(z_{1}\right), f_{2}=\bar{c} g_{2}\left(z_{2}\right)+c_{o}$.

TYPE 4. $f, g$ satisfy the following condition. There exist analytic functions $a_{1}, a_{2}, b_{1}, b_{2}$ and constants $c_{1}, c_{2}, c_{3}$, such that

$$
f_{1}=c_{1} a_{1}\left(z_{2}\right)+c_{2} b_{1}\left(z_{1}\right), f_{2}=\overline{c_{1}} a_{2}\left(z_{2}\right)+\overline{c_{2}} b_{2}\left(z_{1}\right)
$$

and

$$
g_{1}=c_{3} a_{1}\left(z_{2}\right)+b_{1}\left(z_{1}\right), g_{2}=\overline{c_{3}} a_{2}\left(z_{2}\right)+b_{2}\left(z_{1}\right) .
$$

If both $a_{1}$ and $a_{2}$ are constant, then exists a constant $c$ such that $f+c g$ is constant.

Lemma 2.1. Suppose $f, g \in L^{\infty}\left(D^{2}, d A\right)$ and $f=f_{1}+\bar{f}_{2}, g=g_{1}+\bar{g}_{2}$, where $f_{i}, g_{i} \in H^{\infty}\left(D^{2}\right)(i=1,2)$. If $f$ and $g$ are one of the above four Types, then $T_{f} T_{g}=T_{g} T_{f}$.

Proof:

(1) If $f, g$ are Type 1. Without loss of generality, we assume

$$
f=f_{1}(z)+\overline{f_{2}\left(z_{2}\right)}, g=g_{1}\left(z_{1}\right) .
$$

Then $T_{f}=T_{f_{1}}+T_{f_{2}}{ }^{*}, T_{g}=T_{g_{1}}$. By $[7], T_{f_{2}}{ }^{*} T_{g_{1}}=T_{g_{1}} T_{f_{2}}{ }^{*}$, so $T_{f} T_{g}=T_{g} T_{f}$.

(2) If $f, g$ are Type 2. Without loss of generality, we assume

$$
f=f_{1}\left(z_{1}\right)+\overline{f_{2}\left(z_{2}\right)}, g=g_{1}\left(z_{1}\right)+\overline{g_{2}\left(z_{2}\right)} .
$$

By [7], $T_{f_{1}} T_{g_{2}}{ }^{*}=T_{g_{2}}{ }^{*} T_{f_{1}}$ and $T_{f_{2}}{ }^{*} T_{g_{1}}=T_{g_{1}} T_{f_{2}}{ }^{*}$, hence $T_{f} T_{g}=T_{g} T_{f}$.

(3) If $f, g$ are Type 3 . Without loss of generality, we assume

$$
f_{1}=c g_{1}(z)+a_{1}\left(z_{2}\right), f_{2}=\bar{c} g_{2}\left(z_{1}\right)
$$

Then

$$
\begin{aligned}
& T_{f_{2}}{ }^{*} T_{g_{1}}+T_{f_{1}} T_{g_{2}}{ }^{*}=c T_{g_{2}}{ }^{*} T_{g_{1}}+c T_{g_{1}} T_{g_{2}}{ }^{*}+T_{a_{1}} T_{g_{2}}{ }^{*}, \\
& T_{g_{2}}{ }^{*} T_{f_{1}}+T_{g_{1}} T_{f_{2}}{ }^{*}=c T_{g_{2}}{ }^{*} T_{g_{1}}+c T_{g_{1}} T_{g_{2}}{ }^{*}+T_{g_{2}}{ }^{*} T_{a_{1}} .
\end{aligned}
$$

Using [7] again, we have $T_{f} T_{g}=T_{g} T_{f}$.

(4) If $f, g$ are Type 4, the proof is analogous. 
THEOREM 2.2. Suppose $f, g \in L^{\infty}\left(D^{2}, d A\right)$ and $f=f_{1}+\bar{f}_{2}, g=g_{1}+\bar{g}_{2}$, where $f_{i}, g_{i} \in H^{\infty}\left(D^{2}\right)(i=1,2)$. Then $T_{f} T_{g}=T_{g} T_{f}$ if and only if

(a) $f$ and $g$ are both analytic on $D^{2}$; or

(b) $f$ and $g$ are both co-analytic on $D^{2}$; or

(c) $f$ or $g$ is a constant function; or

(d) $f$ and $g$ are one of the above four Types.

PROOF: By Lemma 2.1, the sufficiency of the Theorem is obvious. Now assume $T_{f} T_{g}=T_{g} T_{f}$. Then

$$
\begin{aligned}
\left\langle T_{f} T_{g} k_{z}, k_{z}\right\rangle & =\left\langle f P\left(g k_{z}\right), k_{z}\right\rangle=\left\langle\left(f_{1}+\overline{f_{2}}\right)\left(g_{1} k_{z}+\overline{g_{2}}(z) k_{z}\right), k_{z}\right\rangle \\
& =\left\langle f_{1} g_{1} k_{z}, k_{z}\right\rangle+\left\langle\overline{f_{2}} g_{1} k_{z}, k_{z}\right\rangle+\overline{g_{2}}(z)\left\langle f_{1} k_{z}, k_{z}\right\rangle+\overline{g_{2}}(z)\left\langle\overline{f_{2}} k_{z}, k_{z}\right\rangle \\
& =f_{1}(z) g_{1}(z)+\left\langle\overline{f_{2}} g_{1} k_{z}, k_{z}\right\rangle+f_{1}(z) \overline{g_{2}}(z)+\overline{f_{2}}(z) \overline{g_{2}}(z) .
\end{aligned}
$$

Analogously, we obtain

$$
\left\langle T_{g} T_{f} k_{z}, k_{z}\right\rangle=f_{1}(z) g_{1}(z)+\left\langle\overline{g_{2}} f_{1} k_{z}, k_{z}\right\rangle+g_{1}(z) \overline{f_{2}}(z)+\overline{g_{2}}(z) \overline{f_{2}}(z) .
$$

Combining the above results we conclude

$$
\left\langle\left(T_{f} T_{g}-T_{g} T_{f}\right) k_{z}, k_{z}\right\rangle=\tilde{u}(z)-u(z)=0,
$$

where $u=\overline{f_{2}} g_{1}-f_{1} \overline{g_{2}}$. By [5, Theorem 3.1] we get $u$ is a 2-harmonic function. Thus

$$
\Delta_{1} u=\Delta_{2} u=0 \text {. }
$$

If $f$ is analytic on $D^{2}$, then for $i=1,2$,

$$
\frac{\partial f}{\partial \bar{z}_{i}}=0, \frac{\partial \bar{f}}{\partial z_{i}}=0, \text { and } \frac{\partial \bar{f}}{\partial \overline{z_{i}}}=\frac{\overline{\partial f}}{\partial z_{i}} .
$$

So for $i=1,2$

$$
0=4 \frac{\partial}{\partial \bar{z}_{i}}\left(\frac{\partial u}{\partial z_{i}}\right)=4\left(\overline{\frac{\partial f_{2}}{\partial z_{i}}} \frac{\partial g_{1}}{\partial z_{i}}-\frac{\partial f_{1}}{\partial z_{i}} \frac{\overline{\partial g_{2}}}{\partial z_{i}}\right) \quad \text { on } D^{2}
$$

Thus we have

$$
\begin{array}{ll}
\overline{\frac{\partial f_{2}}{\partial z_{1}}} \frac{\partial g_{1}}{\partial z_{1}}=\frac{\partial f_{1}}{\partial z_{1}} \frac{\overline{\partial g_{2}}}{\partial z_{1}} & \text { on } D^{2} \\
\overline{\frac{\partial f_{2}}{\partial z_{2}}} \frac{\partial g_{1}}{\partial z_{2}}=\frac{\partial f_{1}}{\partial z_{2}} \frac{\overline{\partial g_{2}}}{\partial z_{2}} & \text { on } D^{2} .
\end{array}
$$

To finish the proof, we consider three cases. Because there are many detailed subcases in each case, we let (1.1), (1.2) and (1.3) denote three different subcases in (1). Analogously, (1.3.1), (1.3.2), (1.3.3) denote three different subcases in (1.3), and so on. 
(1) Suppose $\frac{\partial g_{1}}{\partial z_{1}}=0$, then $g_{1}=g_{1}\left(z_{2}\right)$. In this case, either $\frac{\partial f_{1}}{\partial z_{1}}=0$ or $\frac{\partial g_{2}}{\partial z_{1}}=0$. (1.1) If $\frac{\partial f_{1}}{\partial z_{1}}=0$, then $f_{1}=f_{1}\left(z_{2}\right)$. By equation (2.4), we get

$$
f_{1}^{\prime} \frac{\overline{\partial g_{2}}}{\partial z_{2}}=g_{1}^{\prime} \frac{\overline{\partial f_{2}}}{\partial z_{2}}
$$

We analysis equation (2.5). (1.1.1) If $g_{1}^{\prime}\left(z_{2}\right)=0$. Then $g_{1}$ is a constant. (1.1.1.1) When $f_{1}^{\prime}\left(z_{2}\right)=0$, then $f$ and $g$ are coanalytic, (b) would hold. (1.1.1.2) When $\frac{\partial g_{2}}{\partial z_{2}}=0$, then $g=c+\overline{g_{2}\left(z_{1}\right)}, f=f_{1}\left(z_{2}\right)+\overline{f_{2}}$. So $f, g$ are Type $1,(d)$ would hold. (1.1.2) If $\frac{\partial g_{2}}{\partial z_{2}}=0$, then $g=g_{1}\left(z_{2}\right)+\overline{g_{2}\left(z_{1}\right)}$. (1.1.2.1) When $g_{1}^{\prime}\left(z_{2}\right)=0$, this case is (1.1.1.2) and (d) would hold. (1.1.2.2) When $\frac{\partial f_{2}}{\partial z_{2}}=0$, then $f=f_{1}\left(z_{2}\right)+\overline{f_{2}\left(z_{1}\right)}$, and $g=g_{1}\left(z_{2}\right)+\overline{g_{2}\left(z_{1}\right)}$. So $f, g$ are Type 2, (d) would hold. Thus we may assume (1.1.3) that neither $g_{1}^{\prime}$ or $\frac{\partial g_{2}}{\partial z_{2}}$ is identically 0 on $D^{2}$. By (2.5) we have

$$
\overline{\frac{\partial f_{2}}{\partial z_{2}} / \frac{\partial g_{2}}{\partial z_{2}}}=\frac{f_{1}^{\prime}}{g_{1}^{\prime}}
$$

Therefore $f_{1}\left(z_{2}\right)=c g_{1}\left(z_{2}\right)+c_{0}, f_{2}(z)=\bar{c} g_{2}(z)+a_{2}\left(z_{1}\right), f, g$ are Type 3 and (d) holds: (1.2) If $\frac{\partial g_{2}}{\partial z_{1}}=0$, then $g=g_{1}\left(z_{2}\right)+\overline{g_{2}\left(z_{2}\right)}$. By (2.4), we have

$$
\frac{\partial f_{1}}{\partial z_{2}} \overline{g_{2}^{\prime}}=\frac{\overline{\partial f_{2}}}{\partial z_{2}} g_{1}^{\prime}
$$

We analysis equation (2.6). (1.2.1) Suppose $g_{1}^{\prime}=0$, then $g=\overline{g_{2}\left(z_{2}\right)}$. (1.2.1.1) When $g_{2} \prime=0$, then $g$ is a constant and (c) holds. (1.2.1.2) When $\frac{\partial f_{1}}{\partial z_{2}}=0$, then $f=f_{1}\left(z_{1}\right)+\overline{f_{2}}$, $g=\overline{g_{2}\left(z_{2}\right)}$, hence $f, g$ are Type 1 and (d) holds. (1.2.2) Suppose $g_{2}=0$, then $g=g_{1}\left(z_{2}\right)+$ c. (1.2.2.1) If $g_{1} \prime=0$, then $g$ is a constant. (1.2.2.2) If $\frac{\partial f_{2}}{\partial z_{2}}=0$, then $f=f_{1}+\overline{f_{2}\left(z_{1}\right)}$, $g=g_{1}\left(z_{2}\right)$. So $f, g$ are Type 1 and (d) would hold. We assume (1.2.3) both $g_{1}^{\prime}$ and $g_{2}^{\prime}$ are not zero. Then by $(2.6)$,

$$
\frac{\partial f_{1} / \partial z_{2}}{g_{1}{ }^{\prime}}=\frac{\overline{\partial f_{2} / \partial z_{2}}}{g_{2}{ }^{\prime}}
$$

Thus there are a constant $c$ and two analytic functions $a_{1}\left(z_{1}\right), a_{2}\left(z_{1}\right)$, such that

$$
f_{1}=c g_{1}\left(z_{2}\right)+a_{1}\left(z_{1}\right), f_{2}=\bar{c} g_{2}\left(z_{2}\right)+a_{2}\left(z_{1}\right) .
$$

Thus $f, g$ are Type 4 and (d) holds.

(2) Suppose $\frac{\partial g_{2}}{\partial z_{1}}=0$, then $g_{2}=g_{2}\left(z_{2}\right)$. (2.1) If $\frac{\partial g_{1}}{\partial z_{1}}=0$, this case is (1.2) and the theorem are proved. (2.2) If $\frac{\partial f_{2}}{\partial z_{1}}=0$, then $f_{2}=f_{2}\left(z_{2}\right)$. By (2.4) we have

$$
\overline{f_{2}^{\prime}} \frac{\partial g_{1}}{\partial z_{2}}=\overline{g_{2}^{\prime}} \frac{\partial f_{1}}{\partial z_{2}} \text {. }
$$


We analysis equation (2.7). (2.2.1) Suppose $g_{2}^{\prime}=0$. If (2.2.1.1) $\frac{\partial g_{1}}{\partial z_{2}}=0$, then

$$
f=f_{1}+\overline{f_{2}\left(z_{2}\right)}, g=g_{1}\left(z_{1}\right) .
$$

Hence $f, g$ are Type 1 and (d) holds. (2.2.1.2) If $f_{2}^{\prime}=0$, then $f, g$ are analytic and (a) would hold. (2.2.2) Suppose $\frac{\partial g_{1}}{\partial z_{2}}=0$. (2.2.2.1) If $g_{2}^{\prime}=0$, then this case is (2.2.1.1) and is proved. (2.2.2.2) If $\frac{\partial f_{1}}{\partial z_{2}}=0$, then

$$
f=f_{1}\left(z_{1}\right)+\overline{f_{2}\left(z_{2}\right)}, g=g_{1}\left(z_{1}\right)+\overline{g_{2}\left(z_{2}\right)} .
$$

Thus $f, g$ are Type 2 and (d) holds. (2.2.3) Suppose both $g_{2}^{\prime}$ and $\frac{\partial g_{1}}{\partial z_{2}}$ are not zero. By (2.7) we get

$$
\frac{\partial f_{1} / \partial z_{2}}{\partial g_{1} / \partial z_{2}}=\frac{\overline{f_{2}^{\prime}}}{g_{2}^{\prime}}
$$

Then there exist constant $c, c_{0}$ and a analytic function $a_{1}\left(z_{1}\right)$, such that

$$
f_{1}=c g_{1}(z)+a_{1}\left(z_{1}\right), f_{2}=\bar{c} g_{2}\left(z_{2}\right)+c_{0} .
$$

Thus $f, g$ are Type 3 and (d) holds.

(3) Suppose both $\frac{\partial g_{1}}{\partial z_{1}}$ and $\frac{\partial g_{2}}{\partial z_{1}}$ are not zero. By (2.3),

$$
\frac{\overline{\partial f_{2} / \partial z_{1}}}{\partial g_{2} / \partial z_{1}}=\frac{\partial f_{1} / \partial z_{1}}{\partial g_{1} / \partial z_{1}}
$$

So there exist a constant $c$ and analytic functions $a_{1}\left(z_{2}\right), a_{2}\left(z_{2}\right)$ such that

$$
f_{1}(z)=c g_{1}(z)+a_{1}\left(z_{2}\right), f_{2}(z)=\bar{c} g_{2}(z)+a_{2}\left(z_{2}\right) .
$$

Combining (2.4), (2.8), we obtain

$$
\overline{a_{2}^{\prime}} \frac{\partial g_{1}}{\partial z_{2}}=a_{1}^{\prime} \frac{\overline{\partial g_{2}}}{\partial z_{2}}
$$

(3.1) Suppose $a_{1}^{\prime}=0$. (3.1.1) If $a_{2}^{\prime}=0$, then there exist constant $a, b$ such that $f=a g+b$. So $f, g$ are Type 4 and (d) holds. (3.1.2) If $\frac{\partial g_{1}}{\partial z_{2}}=0$, then

$$
f_{1}=c g_{1}\left(z_{1}\right)+c_{1}, f_{2}=\bar{c} g_{2}(z)+a_{2}\left(z_{2}\right) \text {. }
$$

So $f, g$ are Type 3 and (d) holds. Similiarly, if (3.2) $a_{2}^{\prime}=0$, then (d) holds. Suppose (3.3) both $a_{1}^{\prime}$ and $a_{2}^{\prime}$ are not zero. By (2.9) we have

$$
\frac{\partial g_{1} / \partial z_{2}}{a_{1}^{\prime}}=\frac{\overline{\partial g_{2} / \partial z_{2}}}{a_{2}^{\prime}}
$$

Thence there are a constant $c_{1}$ and two analytic functions $b_{1}\left(z_{1}\right)$ and $b_{2}\left(z_{1}\right)$ such that

$$
g_{1}=c_{1} a_{1}\left(z_{2}\right)+b_{1}\left(z_{1}\right), g_{2}=\overline{c_{1}} a_{2}\left(z_{2}\right)+b_{2}\left(z_{1}\right) .
$$

By (2.8) and $(2,10), f, g$ are Type 4 and (d) would hold. 


\section{REFERENCES}

[1] S. Axler and Ž. Čučković, 'Commuting Toeplitz operators with harmonic symbols', Integral Equations Operator Theory 14 (1991), 1-12.

[2] S. Axler, Ž. Čučković and N.V.Rao, 'Commutants of analytic Toeplitz operators on the Bergman space', Proc. Amer. Math. Soc. 128 (2000), 1951-1953.

[3] A. Brown and P.R. Harmols, 'Algebraic properties of Toeplitz operators', J. Reine Angew. Math. 213 (1964), 89-102.

[4] S.G. Krantz, Function theory of several complex variables (J. Wiley and Sons, New York, 1982).

[5] J. Lee, 'An invariant value property in the polydisc', Ilinois J. Math. 42 (1998), 406-419.

6] W. Rudin, Function theory in polydiscs (W.A. Benjamin, New York, Amsterdam, 1969).

[7] S. Sun and D. Zheng, 'Toeplitz operators on the polydisk', Proc. Amer. Math. Soc. 124 (1996), 3351-3356.

Department of Applied Mathematics

Dalian University of Technology

Dalian, 160024

China

e-mail: yufeng_lu@online.ln.cn 